\title{
Neutrophil Elastase Damages the Pulmonary Endothelial Glycocalyx in Lipopolysaccharide- Induced Experimental Endotoxemia
}

Kodai Suzuki, ${ }^{*}$ Hideshi Okada, ${ }^{*}$ Genzou Takemura, ${ }^{\dagger}$ Chihiro Takada, ${ }^{*}$ Ayumi Kuroda, ${ }^{*}$ Hirohisa Yano, ${ }^{*}$ Ryogen Zaikokuji, ${ }^{* \dagger}$ Kentaro Morishita, ${ }^{*}$ Hiroyuki Tomita, ${ }^{\S}$ Kazumasa Oda, ${ }^{*}$ Saori Matsuo, ${ }^{*}$ Akihiro Uchida, ${ }^{*}$ Tetsuya Fukuta, ${ }^{*}$ So Sampei, * Nagisa Miyazaki, ${ }^{\dagger}$ Tomonori Kawaguchi, ${ }^{*}$ Takatomo Watanabe, ${ }^{\top}$ Takahiro Yoshida, ${ }^{*}$ Hiroaki Ushikoshi, ${ }^{*}$ Shozo Yoshida, * Yoichi Maekawa, ${ }^{* * *}$ and Shinji Ogura*

From the Departments of Emergency and Disaster Medicine, * Tumor Pathology, ${ }^{\S}$ and Parasitology and Infectious Diseases, "Gifu University Graduate School of Medicine, Gifu; the Department of Internal Medicine, ${ }^{\dagger}$ Asahi University School of Dentistry, Mizuho, Gifu; the Laboratory of Molecular Biology, ${ }^{\ddagger}$ Department of Biofunctional Analysis, Gifu Pharmaceutical University, Gifu; the Department of Clinical Laboratory, "Gifu University Hospital, Gifu; and the Domain of Integrated Life Systems, ** Center for Highly Advanced Integration of Nano and Life Sciences (G-CHAIN), Gifu University, Gifu, Japan

Accepted for publication

May 1, 2019.

Address correspondence to Hideshi Okada, M.D., Ph.D.,

Department of Emergency and Disaster Medicine, Gifu University Graduate School of Medicine, Yanagido 1-1, Gifu 501-1194, Japan. E-mail: hideshi@gifu-u.ac.jp.

\begin{abstract}
Neutrophil elastase (NE) is necessary for effective sterilization of phagocytosed bacterial and fungal pathogens; however, NE increases alveolocapillary permeability and induces proinflammatory cytokine production in sepsis-induced acute respiratory distress syndrome. Under septic conditions, the pulmonary endothelial glycocalyx covering on the healthy endothelium surface is injured, but the contribution of NE to this injury remains unknown. Our aim was to examine whether NE-induced pulmonary endothelial injury is associated with endotoxemia. Lipopolysaccharide (LPS; $20 \mathrm{mg} / \mathrm{kg}$ ) was injected intraperitoneally into 9- to 12-week-old granulocyte colony-stimulating factor knockout (G-CSFKO) mice, which harbor few neutrophils, and littermate control mice; in a second assay, mice were injected with the NE-inhibitor sivelestat $(0.2 \mathrm{mg} / \mathrm{kg})$ at 3, 6, 9, and 12 hours after LPS administration. Subsequently, vascular endothelial injury was evaluated through ultrastructural analysis. At 48 hours after LPS injection, survival rate was more than threefold higher among G-CSFKO than control mice, and degradation of both thrombomodulin and syndecan-1 was markedly attenuated in G-CSFKO compared with control mice. Ultrastructural analysis revealed attenuated vascular endothelial injury and clear preservation of the endothelial glycocalyx in G-CSFKO mice. Moreover, after LPS exposure, survival rate was approximately ninefold higher among sivelestat-injected mice than control mice, and sivelestat treatment potently preserved vascular endothelial structures and the endothelial glycocalyx. In conclusion, NE is associated with pulmonary endothelial injury under LPS-induced endotoxemic conditions. (Am J Pathol 2019, 189: 1526-1535; https://doi.org/10.1016/j.ajpath.2019.05.002)
\end{abstract}

Sepsis is defined as serious organ dysfunction caused by an uncontrollable host response to infection. ${ }^{1}$ Among the multiple organ dysfunctions that occur, the development of acute respiratory distress syndrome (ARDS) is associated with short- and long-term morbidity, mortality, prolonged hospitalization, and high costs. ${ }^{2}$ ARDS pathogenesis was previously reported to involve the degradation of the endothelial glycocalyx, which serves as an epithelial-cell barrier. ${ }^{3,4}$ Furthermore, we recently reported that glycocalyx disruption participated in microvascular endothelial injury that is distinctive of sepsis-induced ARDS; we found that the endothelial glycocalyx in the lung was obviously disrupted by lipopolysaccharide (LPS).

\footnotetext{
Supported in part by the Ministry of Education, Science and Culture of Japan grants-in-aid for scientific research 18K08914 (K.S.), 17K11569 (S.O.), 16H05497 (H.O.), 16K20381 (K.S.), and 15K10973 (T.Y.). Sivelestat, a neutrophil elastase competitive antagonist, was gifted from Ono Pharmaceutical Co., Ltd., Osaka, Japan.

Disclosures: None declared.
} 
The endothelial glycocalyx plays an important role in regulating microvascular homeostasis by maintaining endothelial permeability and microvascular tone, preserving an oncotic gradient across the endothelial barrier, and modulating adhesion/migration of leukocytes. ${ }^{6}$ Degradation of the endothelial glycocalyx facilitates the inflow of fluid, including lots of protein and macromolecules into the alveolar space, ${ }^{7}$ and exacerbated pulmonary vascular permeability is associated with endothelial glycocalyx degradation under septic conditions. ${ }^{5}$ Pulmonary endothelial glycocalyx injury increases the availability of endothelial surface adhesion molecules to circulating microspheres and contributes to neutrophil adhesion under septic conditions. ${ }^{3}$

Neutrophils are regarded to play a crucial role in ARDS aggravation; neutrophil activation and transmigration constitute a hallmark event in the progression of this disease. ${ }^{8}$ Moreover, neutrophil elastase (NE), which is necessary for effective sterilization of phagocytosed bacterial and fungal pathogens, ${ }^{9-11}$ is known to increase alveolocapillary permeability, induces the production of proinflammatory cytokines, and enhances neutrophil migration. ${ }^{12-14}$ However, to our knowledge, no study has investigated whether neutrophils and NE injure the pulmonary endothelial glycocalyx under septic conditions. Therefore, we hypothesized that neutrophils and NE injure the pulmonary endothelial glycocalyx during sepsis, and to test this we evaluated the condition of the pulmonary endothelial glycocalyx after LPS administration in granulocyte colonystimulating factor knockout (G-CSFKO) mice and in wildtype (WT) mice treated with the NE inhibitor sivelestat.

\section{Methods}

\section{In Vivo Animal Studies}

This study conformed to the Guide for the Care and Use of Laboratory Animals ${ }^{15}$ and was approved by the Institutional Animal Research Committee of Gifu University (Gifu, Japan). Male G-CSFKO mice (B6; 129P2-Csf3tm1Ard/J) were purchased from The Jackson Laboratory (Bar Harbor, ME) and bred and genotyped as described ${ }^{16}$; 9- to 12-week-old GCSFKO and littermate control mice were used in this study. After 16 hours of starvation, mice were intraperitoneally administered LPS (20 mg/kg; MilliporeSigma, Burlington, MA), and in certain assays the NE-inhibitor sivelestat (gifted from Ono Pharmaceutical Co., Ltd., Osaka, Japan) was intraperitoneally administrated at 3, 6, 9, and 12 hours after LPS injection. Survival rate was determined at $12,24,36$, and 48 hours after LPS administration, and surviving mice were sacrificed, and lung specimens were collected.

\section{Serum Preparation and Enzyme-Linked Immunosorbent Assay}

Blood samples were collected from the maxillary artery, allowed to clot at room temperature for 2 hours, and centrifuged at $2000 \times g$ for $4^{\circ} \mathrm{C}$ for 20 minutes. The supernatant was collected as the serum for measuring $\mathrm{NE}$ levels by using enzyme-linked immunosorbent assay quantitation kits for mouse NE (E-EL-M0444; Elabscience, Houston, TX).

\section{Flow Cytometric Analysis of Peripheral Neutrophils}

Before and at 6 hours after LPS administration, blood samples were collected in the same manner. Samples were prepared as previously described. ${ }^{17}$

Cells were stained with the following antibodies (BioLegend, San Diego, CA) for 5 minutes at room temperature: anti-mouse CD45-peridinin chlorophyll protein complex (clone: 30-F11), anti-mouse CD11b-phycoerythrin (clone: M1/70), anti-mouse Ly6G-fluorescein isothiocyanate (clone: 1A8), and anti-mouse F4/80-Alexa Fluor 647 (clone: BM8). Neutrophils were defined as the $\mathrm{CD} 45^{+} \mathrm{CD} 11 \mathrm{~b}^{+} \mathrm{Ly}_{6 \mathrm{G}}{ }^{+} \mathrm{F} 4 / 80^{-}$white blood cell population. Flow cytometry was performed on a BD FACSCalibur instrument (Becton and Dickinson Company, Franklin Lakes, $\mathrm{NJ}$ ), and data were analyzed with FlowJo software version 10.5.3 (TreeStar LLC, Ashland, OR).

\section{Histopathologic Scoring}

After deparaffinization, sections were cut ( $4 \mu \mathrm{m}$ thick), lung tissues were counterstained with hematoxylin and eosin and were scored by a certificated pathologist as follows for neutrophilic infiltration: 1 , absent to rare solitary neutrophils; 2 , detectable extravasated neutrophils observed as small loose cellular accumulates in one to a few airways and/or alveoli; 3 , detectable extravasated neutrophils observed as loose to compact cellular accumulates in multiple to coalescing airway and/or alveoli with some effacement of lung architecture; and 4 , detectable extravasated neutrophils observed as compact cellular accumulates effacing most adjacent pulmonary structure. Pulmonary edema was scored as 1, absent; 2, detectable seroproteinaceous fluid in one to a few alveoli; and 3 , seroproteinaceous fluid filling alveoli in a multifocal to coalescing pattern in lung. ${ }^{18}$

\section{Immunohistochemistry}

Lung sections incubated with primary antibodies against the neutrophil surface marker Gr-1 (ab8592; Abcam, Cambridge, UK) and the endothelial-injury surface marker thrombomodulin (ab6980; Abcam). Sections were immunostained with the Vectastain Elite ABC system (Vector Laboratories, Burlingame, CA) as described. ${ }^{5,19}$ A trained pathologist counted the incidence of thrombomodulinpositive cells in the total endothelial cells. The cell count was performed on five randomly chosen high-power field (HPF) in each section. The number of endothelial cells evaluated was $253 \pm 21$ cells per lung. 

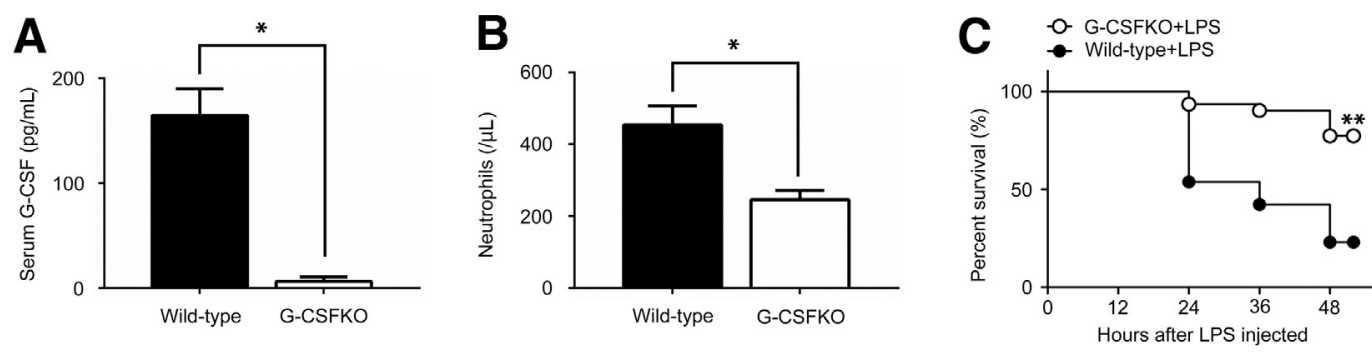

D
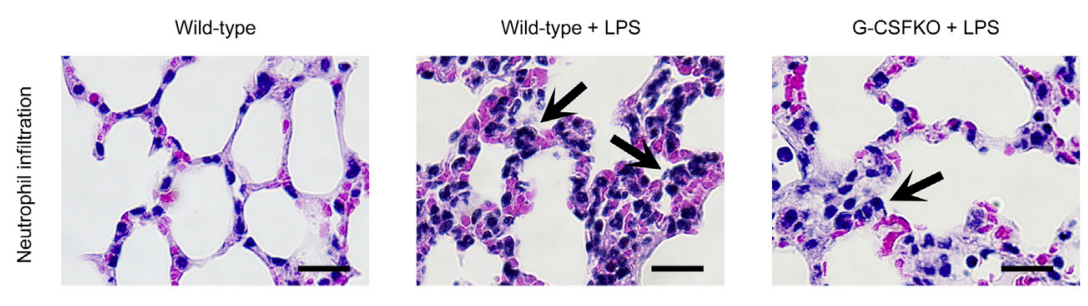

E
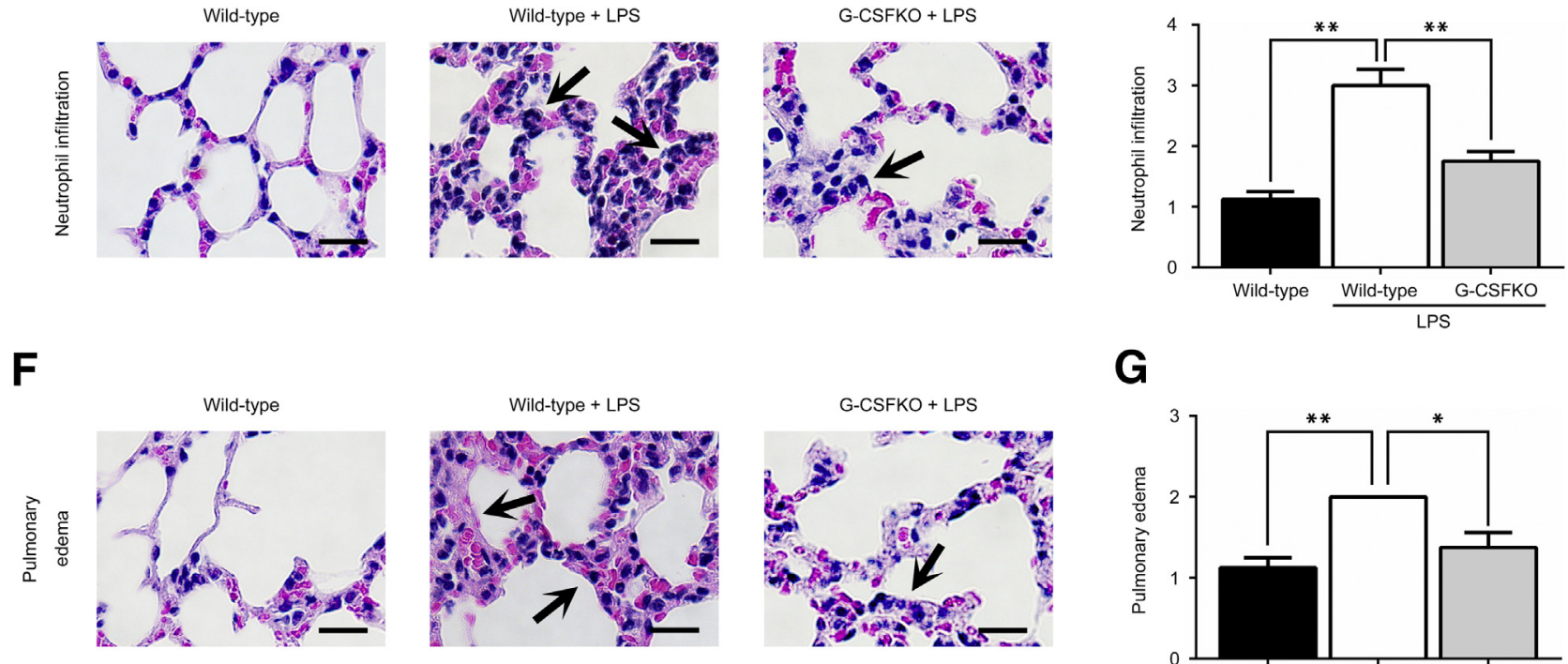

G

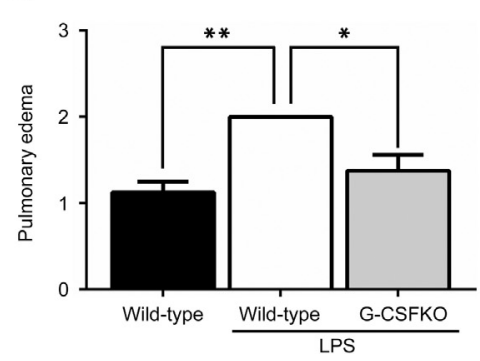

Figure 1 Granulocyte colony-stimulating factor knockout (G-CSFK0) mice resist lipopolysaccharide (LPS)-induced neutrophil invasion. A: Serum G-CSF level in untreated wild-type and G-CSFKO mice was measured with enzyme-linked immunosorbent assay. B: Peripheral neutrophils $\left(\mathrm{CD} 45^{+} \mathrm{CD} 11 \mathrm{~b}^{+} \mathrm{Ly} 6 \mathrm{G}^{+} \mathrm{F} 4 / 80^{-}\right)$in untreated wild-type and G-CSFKO mice were quantified with flow cytometry. C: Kaplan-Meier survival curves for wild-type mice and G-CSFKO mice after LPS administration. D: Hematoxylin and eosin-stained lung tissues. Arrows indicate neutrophil infiltration. E: Graphs of the histologic scoring of lung injury about neutrophil filtration. F: Hematoxylin and eosin-stained lung tissues. Arrows indicate edema. Graphs of the histologic scoring of lung injury about pulmonary edema. Data are expressed as means \pm SEM. $n=5$ mice per group (A); $n=6$ mice per group (B); $n=26$ wild-type mice (C); $n=31 \mathrm{G}$-CSFKO mice (C). ${ }^{*} P<0.05,{ }^{*} P P<0.01$ versus wild-type. Scale bars: $20 \mu \mathrm{m}$ (D and $\left.\mathbf{F}\right)$.

\section{Western Blot Analysis}

Tissue lysate preparation and electrophoresing were performed as previously described..$^{5}$ The membranes were probed with antibodies against thrombomodulin (Abcam), syndecan-1 (ab34164; Abcam), or $\alpha$-tubulin (sc-5546; Santa Cruz Biotechnology, Dallas, TX), and then immunoreactive bands were visualized with enhanced chemiluminescence (GE Healthcare UK Ltd., Little Chalfont, Buckinghamshire, UK), and signal intensities were quantified (as arbitrary units) with the use of ImageJ software version 1.51j8 (NIH, Bethesda, MD; http://imagej.nih.gov/ij). Western blot analysis was performed on four samples from each group.

\section{Electron Microscopy}

Electron microscopy analysis of the endothelial glycocalyx was performed as previously described. ${ }^{5,19}$ Briefly, mice were anesthetized and then perfused with a solution composed of $2 \%$ glutaraldehyde, $2 \%$ sucrose, $0.1 \mathrm{~mol} / \mathrm{L}$ sodium cacodylate buffer ( $\mathrm{pH} 7.3$ ), and $2 \%$ lanthanum nitrate, at a steady flow-rate of $1 \mathrm{~mL} / \mathrm{min}$, through a cannula placed in the left ventricle. After the mice were sacrificed, lung samples were fixed in a solution without glutaraldehyde and then washed in alkaline $(0.03 \mathrm{~mol} / \mathrm{L} \mathrm{NaOH}) 2 \%$ sucrose solution. The freeze-fracture method was used to prepare samples for scanning electron microscopy (S-4800; Hitachi High-Technologies Global, Tokyo, Japan). To prepare samples for transmission electron microscopy (TEM), specimens were embedded in epoxy resin and then ultrathin (90-nm) sections were generated, stained with uranyl acetate and lead citrate, and subjected to TEM analysis (HT-7700, Hitachi High-Technologies Global, Tokyo, Japan). To prepare samples for conventional electron microscopy, $2.5 \%$ glutaraldehyde in $0.1 \mathrm{~mol} / \mathrm{L}$ phosphate buffer $(\mathrm{pH}$ 7.4) without lanthanum nitrate was used as the fixative.

\section{Data Analysis}

Data are presented as means \pm SEM. Two-tailed $t$-test was used for comparing two groups, and survival data were 
analyzed with the log-rank test; $P<0.05$ was considered significant. All calculations were performed with Prism software version 7.02 (GraphPad, La Jolla, CA).

\section{Results}

Neutrophils and NE in G-CSFKO Mice under LPS Administration

Analysis of the enzyme-linked immunosorbent assay revealed serum G-CSF deficiency, and flow cytometric analysis revealed neutropenia in G-CSFKO mice (Figure 1, $\mathrm{A}$ and $\mathrm{B}$ ), confirming the original report. ${ }^{20}$

To produce LPS-induced experimental endotoxemia model mice, $20 \mathrm{mg} / \mathrm{kg}$ LPS was injected intraperitoneally to 9- to 12-week-old G-CSFKO and littermate control male mice. At 48 hours after LPS administration, the survival rate of G-CSFKO mice $(77 \%, 24$ of 31) was markedly higher $(P<0.05)$ than that of control WT mice $(23 \%, 6$ of 26$)$ (Figure 1C).

To determine pulmonary injury 48 hours after LPS injection, a scoring system was used (Figure $1, \mathrm{D}-\mathrm{G}$ ). ${ }^{18}$ After LPS administration, levels of neutrophil infiltration and pulmonary edema increased compared with before LPS injection. Conversely, G-CSFKO mice had a significant decrease in neutrophil infiltration and pulmonary edema.

In WT mice, serum NE concentration was increased at 6 hours after LPS administration and then the level decreased gradually; by comparison, serum NE was considerably lower before and at 6 and 12 hours after LPS injection in GCSFKO mice (Figure 2A). Furthermore, peripheral neutrophil numbers were markedly lower in G-CSFKO mice than WT mice, both before and at 6 hours after LPS administration, the time at which serum NE concentration peaked (Figure 2B), and at 6 hours after LPS injection substantially fewer Gr-1-positive cells were present in the pulmonary tissues of G-CSFKO mice ( $24 \pm 4$ cells/HPF) than WT mice (104 \pm 4 cells/HPF) (Figure 2, C and D).

\section{Pulmonary Endothelial Injury and Endothelial Glycocalyx Injury Are Attenuated in G-CSFKO Mice}

To investigate pulmonary endothelial injury, the localization of thrombomodulin, an endothelial-injury marker, was examined. Immunohistochemical analysis revealed that thrombomodulin was expressed on pulmonary capillaries in both WT and G-CSFKO mice under sham treatment (Figure 3, A and B), but little thrombomodulin was expressed in WT mice after LPS administration. LPS treatment markedly reduced thrombomodulin expression in WT mice but not in G-CSFKO mice (Figure 3, A-D). In accordance with these results, the expression of syndecan-1, an endothelial glycocalyx injury marker, was considerably lower in LPS-treated WT mice than in untreated WT mice (Figure 3, E and F). Conversely, syndecan-1 expression in LPS-treated G-CSFKO mice was not different from that in
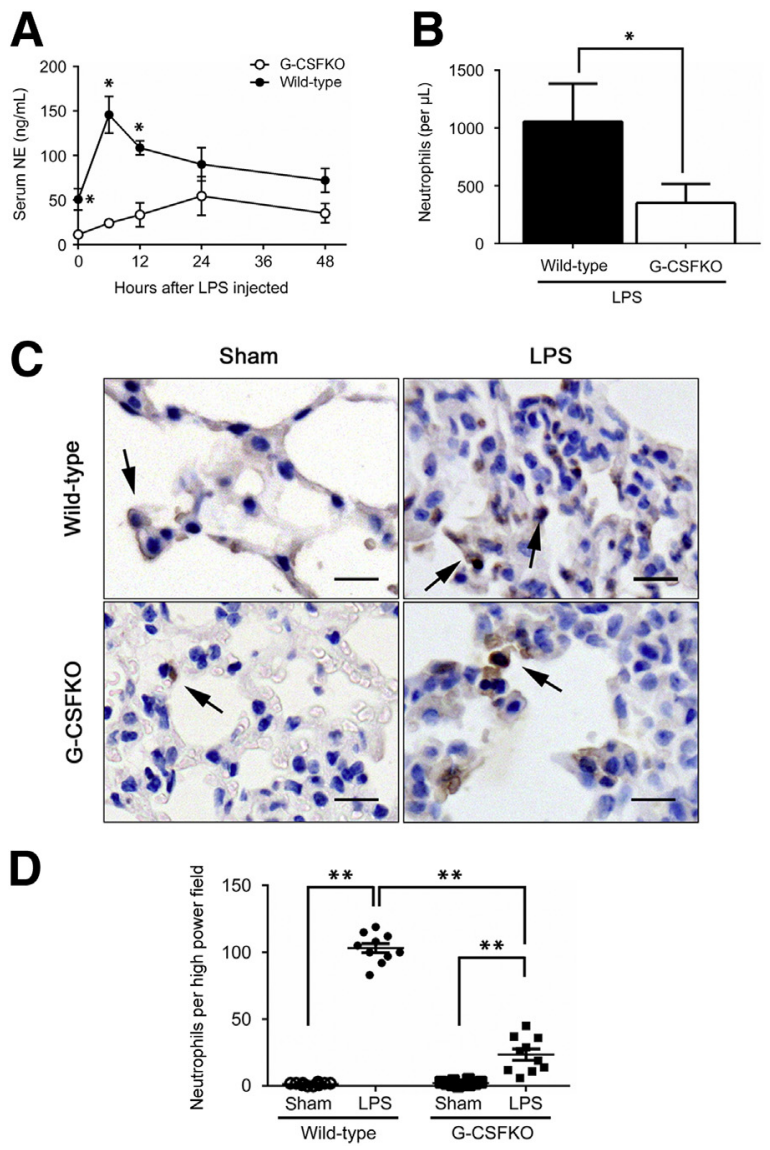

Figure 2 Granulocyte colony-stimulating factor knockout (G-CSFKO) mice exhibited diminished neutrophil accumulation under endotoxemic conditions. A: Serum neutrophil elastase (NE) was measured in mice by using enzyme-linked immunosorbent assay. B: At 6 hours after lipopolysaccharide (LPS) administration, the numbers of peripheral neutrophils $\left(C_{4} 5^{+}\right.$CD11b ${ }^{+}$Ly6G $\left.^{+} \mathrm{F}_{4} / 80^{-}\right)$in wild-type and G-CSFKO mice were determined with flow cytometry. C: Extravasated neutrophils were immunostained for Gr-1 in lung sections from G-CSFKO and wild-type mice, both before and at 6 hours after LPS administration. Arrows indicate Gr1-positive cells. D: The neutrophils were quantified as numbers of cells per high-power field. Data are expressed as means \pm SEM. $n=4$ mice per group (A); $n=6$ mice per group (B); $n=10$ mice per group (D). ${ }^{*} P<0.05,{ }^{*} P<0.01$ versus wild-type. Scale bars: $20 \mu \mathrm{m}(\mathbf{C})$.

untreated WT mice (Figure 3, E and F). These results suggest that pulmonary endothelial injury and endothelial glycocalyx injury in LPS-treated G-CSFKO mice were diminished relative to those in LPS-treated WT mice.

\section{Pulmonary Endothelial Injury Are Attenuated in G-CSFKO Mice under Endotoxemic Conditions}

The ultrastructure of the endothelium and endothelial glycocalyx was analyzed with electron microscopy. Conventional scanning electron microscopy results showed that pulmonary capillaries were of the continuous type, characterized by the presence of an uninterrupted endothelium and a continuous basal lamina, in untreated WT and G-CSFKO mice (Figure 4A). Conventional TEM revealed that after 


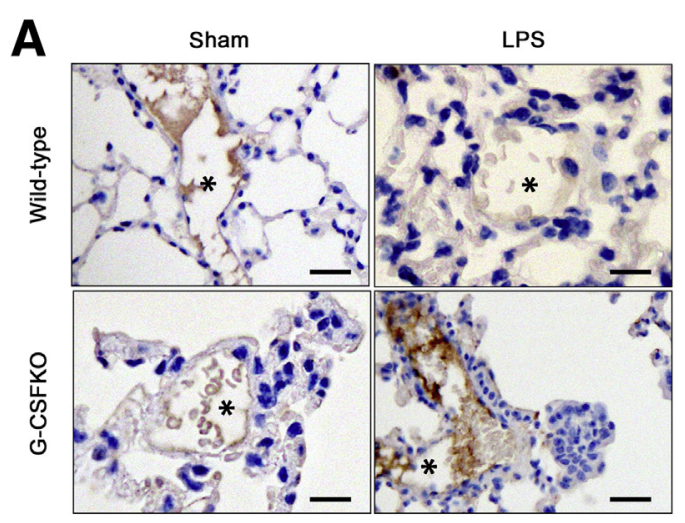

B

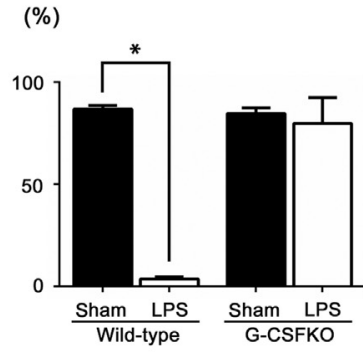

Figure 3 Pulmonary endothelial injury and endothelial glycocalyx injury were attenuated in granulocyte colony-stimulating factor knockout (G-CSFKO) mice. A: Pulmonary endothelial cells were immunostained for thrombomodulin (TM) in lung sections from G-CSFKO and wild-type mice, before and at 48 hours after lipopolysaccharide (LPS) administration. Asterisk indicates a pulmonary vessel. B: Incidence of thrombomodulinpositive cells within all endothelial cells. $\mathbf{C}-\mathbf{F}$ : Western blot analysis for TM (C) and syndecan-1 (E), with $\alpha$-tubulin used as a loading control, in lung homogenates from G-CSFKO and wild-type mice, before and at 48 hours after LPS administration; TM (D) and syndecan-1 (F) levels were normalized relative to $\alpha$-tubulin levels Data are expressed as means \pm SEM. $n=4$ mice per group

E

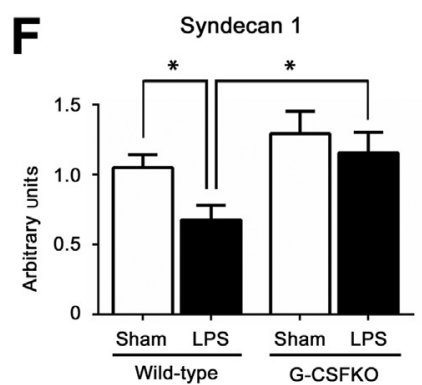
Scale bars: $20 \mu \mathrm{m}(\mathbf{A})$.

LPS injection the endothelial wall became edematous, but the extent was lesser in G-CSFKO mice than in WT mice (Figure 4B).

Scanning electron microscopy and TEM analyses performed with lanthanum revealed an endothelial glycocalyx that featured a moss-like structure on the surface of the vascular endothelium in both WT and G-CSFKO mice under normal conditions; however, after LPS injection, the endothelial glycocalyx was peeled away in WT mice, and this was again attenuated in G-CSFKO mice (Figure 4, C-P).

\section{Sivelestat Increases Survival Rate under Endotoxemia}

Next, to determine the contribution of NE in septic mice, 0.2 $\mathrm{mg} / \mathrm{kg}$ sivelestat was injected intraperitoneally into mice at 3, 6, 9, and 12 hours after LPS administration. Sivelestat forms the acyl complex with an active region of the NE and inhibits NE activity. ${ }^{21}$ At 48 hours after LPS treatment, the survival rate was significantly higher $(P<0.05)$ among sivelestat-injected mice $(82 \%, 22$ of 27$)$ than control mice (9\%, 3 of 32) (Figure 5A).

In sivelestat-treated mice, the levels of neutrophil infiltration and pulmonary edema were attenuated compared with untreated mice 48 hours after LPS injection (Figure 5, $\mathrm{B}-\mathrm{D})$.

At 24 hours after LPS administration, serum NE concentration was markedly lower $(P<0.05)$ in sivelestat-treated mice than in control (saline-treated) mice (Figure 5E). Moreover, the Gr-1-positive cell numbers within pulmonary tissues were obviously increased $(P<0.01)$ in LPS-injected mice $(108 \pm 4$ cells/HPF) compared with that in untreated mice ( $12 \pm 1$ cells/HPF), but there were no significant differences between the numbers of peripheral neutrophils in untreated and sivelestat-treated mice (Figure $5 \mathrm{~F}$ ) or the numbers of extravasated Gr-1-positive cells in the lung sections from these mice at 6 hours after LPS administration (Figure 5, G and $\mathrm{H}$ ).

\section{Sivelestat Attenuates Pulmonary Endothelial Injury under Endotoxemia}

Finally, to examine the protective effect of sivelestat treatment against pulmonary endothelial injury and endothelial glycocalyx injury under endotoxemia, thrombomodulin and syndecan-1 expression was analyzed. In sivelestat-treated mice, thrombomodulin was detected on pulmonary 

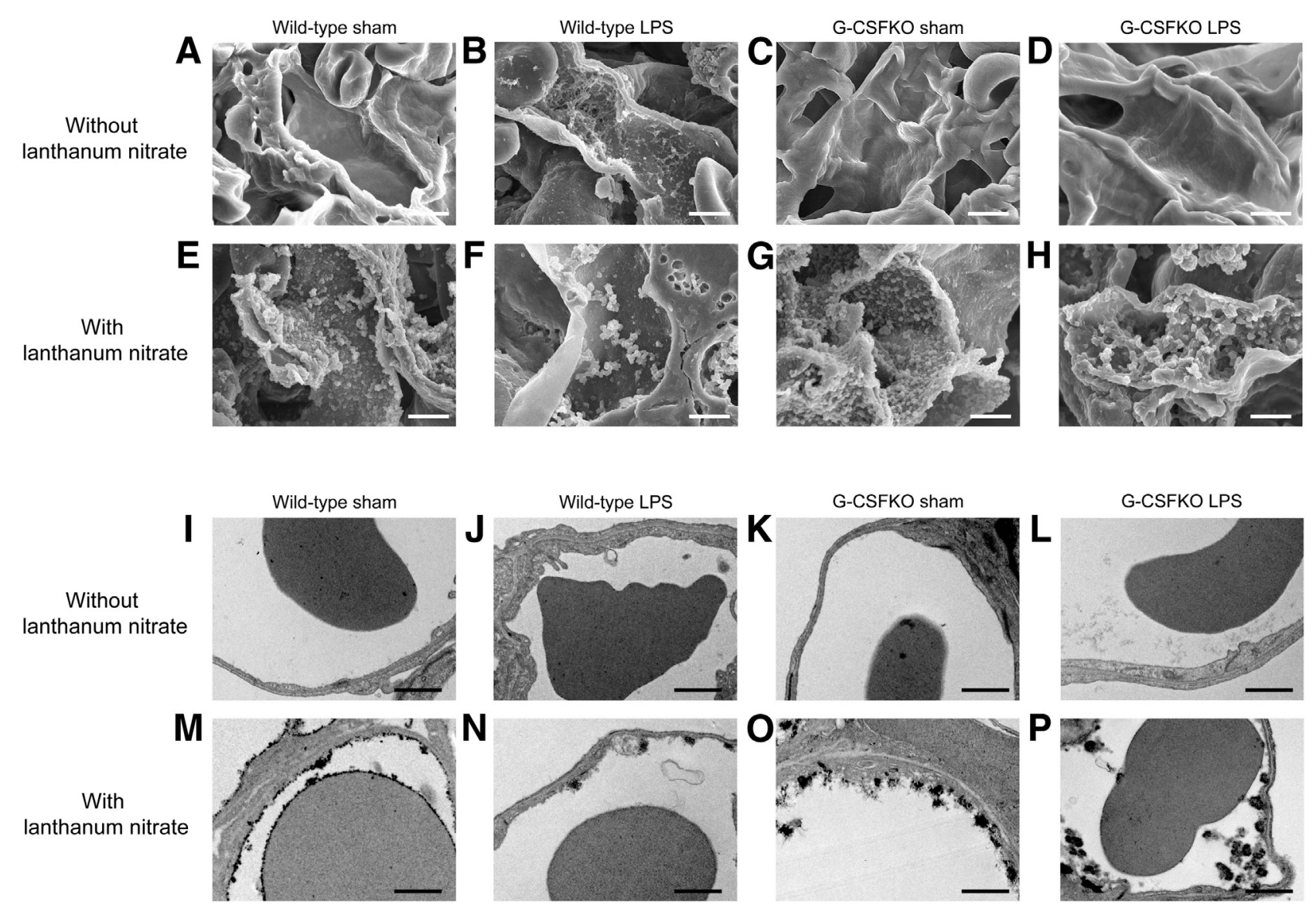

Figure 4 Pulmonary endothelial injury and endothelial glycocalyx injury were attenuated to a greater extent morphologically in lipopolysaccharide (LPS)treated granulocyte colony-stimulating factor knockout (G-CSFKO) mice than in LPS-treated wild-type mice. A-D and I-L: Conventional imaging (without lanthanum nitrate) of pulmonary endothelium through scanning $(\mathbf{A}-\mathbf{D})$ and transmission $(\mathbf{I}-\mathbf{L})$ electron microscopy in untreated wild-type mice (wild-type sham; A and I), LPS-treated wild-type mice (wild-type LPS; $\mathbf{B}$ and $\mathbf{J}$ ), untreated G-CSFKO mice (G-CSFKO sham; C and K), and LPS-treated G-CSFKO mice (G-CSFKO LPS; $\mathbf{D}$ and $\mathbf{L}$ ). $\mathbf{E}-\mathbf{H}$ and $\mathbf{M}-\mathbf{P}$ : Glycocalyx imaging (with lanthanum nitrate) through scanning $(\mathbf{E}-\mathbf{H})$ and transmission (M-P) electron microscopy in untreated wild-type mice (wild-type sham; $\mathbf{E}$ and $\mathbf{M}$ ), LPS-treated wild-type mice (wild-type LPS; $\mathbf{F}$ and $\mathbf{N}$ ), untreated G-CSFKO mice (G-CSFKO sham; $\mathbf{G}$ and $\mathbf{0}$ ), and LPS-treated G-CSFKO mice (G-CSFKO LPS; H and P). Scale bars: $2 \mu \mathrm{m}(\mathbf{A}-\mathbf{H}) ; 1 \mu \mathrm{m}(\mathbf{I}-\mathbf{P})$.

capillaries after LPS administration in the same manner as in untreated control mice (Figure 5, I and J); however, quantitative Western blot analyses showed that thrombomodulin and syndecan-1 expression in lung tissue after LPS administration was enhanced in sivelestat-injected mice compared with that in saline-injected mice (Figure 5, K-N). Notably, ultrastructural analysis revealed that after LPS administration, the endothelial surface was less edematous and the endothelial glycocalyx was preserved to a greater extent in sivelestat-treated WT mice than in saline-treated WT mice (Figure 6). These results suggest that pulmonary endothelial injury and endothelial glycocalyx injury were ameliorated in sivelestat-treated mice compared with that in untreated mice after LPS injection.

\section{Discussion}

This study revealed that G-CSFKO mice and sivelestattreated WT mice showed attenuated pulmonary endothelial glycocalyx injury after LPS administration compared with LPS-treated WT mice. Our findings indicate that the absence of G-CSF and the inhibition of NE critically contribute to glycocalyx protection.

The inner surface of vascular endothelial cells is coated with a glycocalyx of membrane-bound macromolecules, including glycoproteins, hyaluronan, sulfated proteoglycans, and plasma proteins that adhere to this surface matrix. $^{22}$ Degradation of the endothelial glycocalyx has been suggested to contribute to ARDS pathogenesis, 3,4 and in a previous report we presented three-dimensional images that revealed exacerbated pulmonary vascular permeability and degradation of the endothelial glycocalyx on the inner surface of endothelial cells under septic conditions. ${ }^{5}$

We presented ultrastructural imaging of the endothelial glycocalyx in the present study. The endothelial glycocalyx forms a continuous structure that allows for vascular homeostasis. Other than confirming the location of the existence of glycocalyx with the use of a lectin staining technique, the resolution of an optical microscope is not enough to accurately detect the structure. We recently reported that the structures of the endothelial glycocalyx differ greatly among the brain, heart, and lung. ${ }^{23}$ In this report, the 

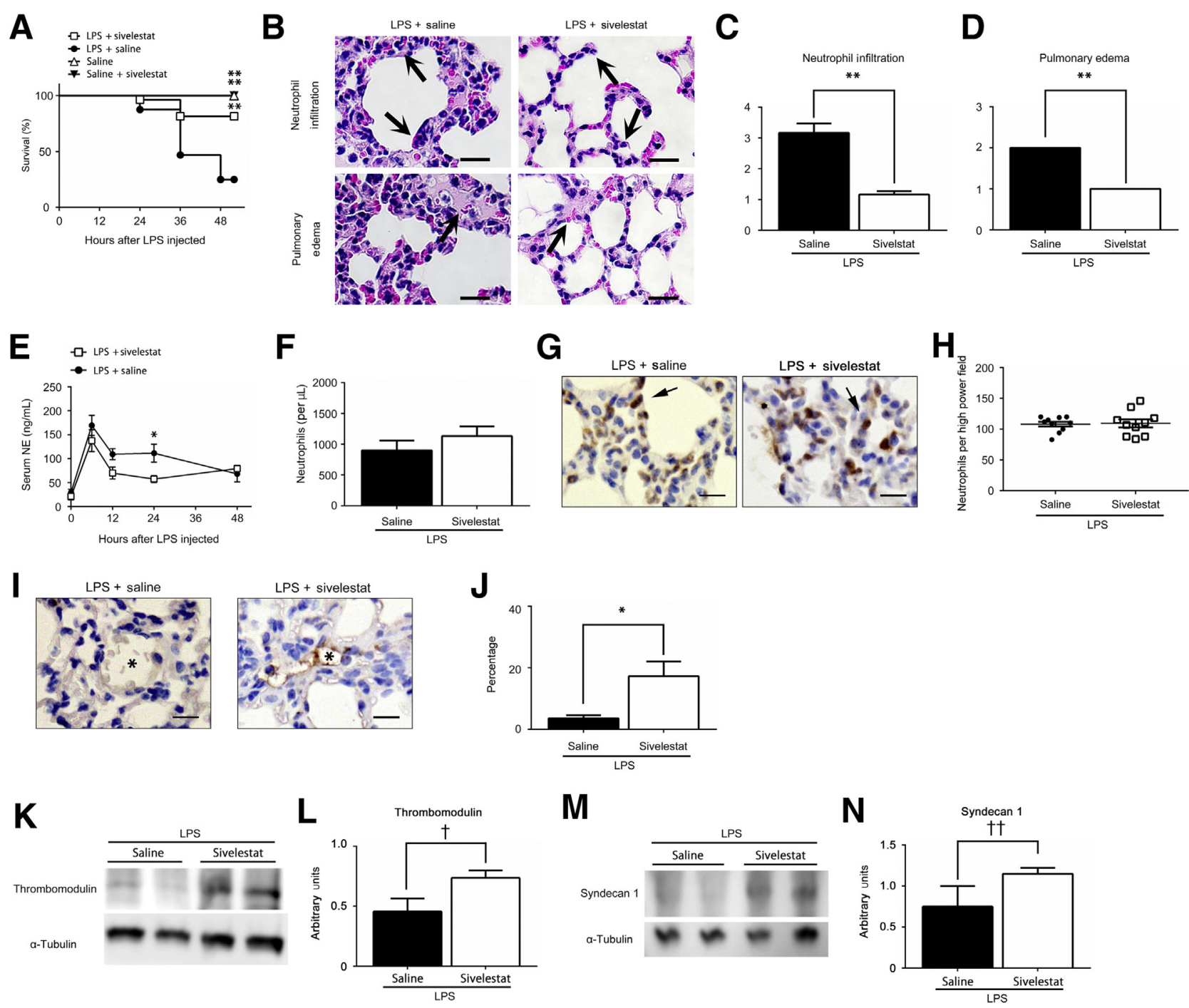

Figure 5 Sivelestat-treat mice resist lipopolysaccharide (LPS)-induced neutrophil invasion. A: Kaplan-Meier survival curves for untreated endotoxemic mice (LPS + saline), sivelestat-treated endotoxemic mice (LPS + sivelestat), saline-treated sham mice (saline), and sivelestat-treated sham mice (saline + sivelestat). B: Hematoxylin and eosin-stained lung tissues. Arrows indicate neutrophils (upper panels) and pulmonary edema (lower panels). C and D: Graphs of the histologic scoring of lung injury, due to neutrophil filtration (C) and pulmonary edema (D). E: Serum neutrophil elastase (NE) levels were measured in mice by using enzyme-linked immunosorbent assay. F: At 6 hours after LPS administration, numbers of peripheral neutrophils $\left(\mathrm{CD}_{5} 5^{+} \mathrm{CD} 11 \mathrm{~b}^{+} \mathrm{Ly}_{6 \mathrm{G}}{ }^{+} \mathrm{F} 4 / 80^{-}\right)$in untreated mice (LPS) and sivelestat-treated endotoxemic mice (sivelestat) were determined with flow cytometry. G: Extravasated neutrophils were immunostained for Gr-1 in lung sections from untreated mice (LPS) and sivelestat-treated endotoxemic mice (sivelestat) at 6 hours after LPS administration. Arrows indicate Gr-1-positive cells. H: The neutrophils were quantified as numbers per high-power field. I: Pulmonary endothelial cells were immunostained for thrombomodulin in lung sections from untreated mice (LPS) and sivelestat-treated endotoxemic mice (sivelestat), both before and at 48 hours after LPS administration. Asterisk indicates a pulmonary vessel. J: Incidence of thrombomodulin (TM)positive cells within all endothelial cells. $\mathbf{K}-\mathbf{N}$ : Western blot analysis for TM (K) and syndecan-1 (M), with $\alpha$-tubulin used as a loading control, in lung homogenates from untreated mice (LPS) and sivelestat-treated endotoxemic mice (sivelestat), both before and at 48 hours after LPS administration; TM and syndecan-1 levels ( $\mathbf{L}$ and $\mathbf{N}$ ) were normalized relative to $\alpha$-tubulin levels. Data are expressed as means \pm SEM. $n=32$ untreated endotoxemic mice (A); $n=27$ sivelestat-treated endotoxemic mice $(\mathbf{A}) ; n=20$ saline-treated sham mice $(\mathbf{A}) ; n=20$ sivelestat-treated sham mice $(\mathbf{A}) ; n=4$ mice per group $(\mathbf{E}, \mathbf{L}$, and $\mathbf{N}) ; n=6$ mice per group $(\mathbf{F}) ; n=5$ mice per group $(\mathbf{H}) .{ }^{*} P<0.05,{ }^{*} P<0.01$ versus LPS + saline; ${ }^{\dagger} P<0.05,{ }^{\dagger \dagger} P<0.01$ versus LPS. Scale bars: $20 \mu \mathrm{m}$ (B, G, and I).

pulmonary capillary glycocalyx is constructed of a thin layer; therefore, it is particularly difficult to detect its structure without ultrastructural imaging. Likewise, in a quantitative assessment of protein expression by Western blot analysis, it is impossible to confirm that the endothelial glycocalyx actually exists on the inner surface of the vascular endothelium. Therefore, we believe that the glycocalyx ultrastructural imaging is a helpful tool in the present study.

Sepsis induces alterations of neutrophil deformability and neutrophil entrapment in lung capillaries; neutrophil entrapment is followed by permeability alterations and 

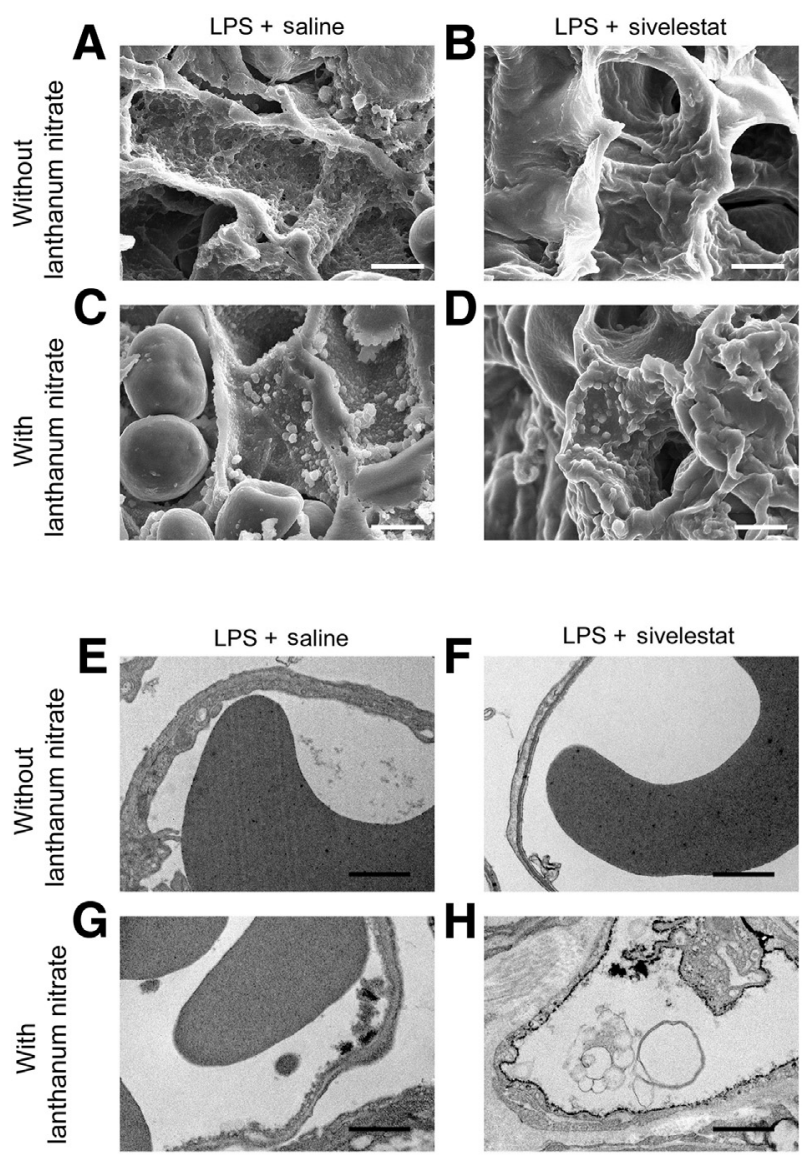

Figure 6 Pulmonary endothelial injury and endothelial glycocalyx injury were attenuated to a greater extent morphologically in sivelestattreated endotoxemic mice than in untreated endotoxemic mice. A, B, E and $\mathbf{F}$ : Conventional imaging (without lanthanum nitrate) of pulmonary endothelium through scanning ( $\mathbf{A}$ and $\mathbf{B}$ ) and transmission ( $\mathbf{E}$ and $\mathbf{F}$ ) electron microscopy in untreated endotoxemic mice [lipopolysaccharide (LPS); $\mathbf{A}$ and $\mathbf{E}$ ] and sivelestat-treated endotoxemic mice (sivelestat; $\mathbf{B}$ and F). C, D, G and H: Glycocalyx imaging (with lanthanum nitrate) through scanning ( $\mathbf{C}$ and $\mathbf{D})$ and transmission ( $\mathbf{G}$ and $\mathbf{H}$ ) electron microscopy in untreated endotoxemic mice (LPS; $\mathbf{C}$ and $\mathbf{G}$ ) and sivelestat-treated endotoxemic mice (sivelestat; D and $\mathbf{H})$. Scale bars: $2 \mu \mathrm{m}(\mathbf{A}-\mathbf{D}) ; 1 \mu \mathrm{m}$ (E-H).

edema formation. ${ }^{24,25}$ In ARDS, a histologic hallmark secondary to sepsis is the recruitment of neutrophils in the lung. ${ }^{26}$ A previous study conducted with the use of sepsis models suggested that mortality was also diminished in granulocyte macrophage-CSFKO mice. ${ }^{27}$

G-CSF, which is produced by monocytes, endothelial cells, and fibroblasts, stimulates neutrophil production and regulates the function and activity of developing and mature neutrophils. ${ }^{28}$ G-CSFKO mice reveal granulocyte and macrophage progenitor cell deficiencies, chronic neutropenia, and impaired neutrophil mobilization. ${ }^{20}$ In G-CSFKO mice, fewer neutrophils are present than in WT mice, even under endotoxemic conditions. Given that endothelial glycocalyx injury after LPS injection was markedly attenuated in G-CSFKO mice compared with that in WT mice, neutrophils might exert harmful effects on the endothelial glycocalyx under septic conditions. However, two explanations can account for the increased survival rate and attenuated endothelial glycocalyx injury in G-CSFKO mice after LPS administration: neutropenia produced a protective effect against endotoxemia in mice; and the amount of neutrophil-secreted mediators was insufficient for causing endothelial injury.

NE was previously reported to play a pivotal role during the pathogenesis of acute lung injury/ARDS ${ }^{26}$ and elevated NE levels were correlated with the severity of lung injury. ${ }^{29-31}$ These reports support our present results. We demonstrated that the pulmonary endothelial glycocalyx injury of sivelestat-treated WT mice was attenuated compared with that of saline-treated WT mice under endotoxemia. Because sivelestat is a competitive antagonist of $\mathrm{NE}$, we suspect that $\mathrm{NE}$ concentrations did not differ substantially between sivelestat-treated and untreated mice in this study. However, a previous clinical study indicated that intravenous sivelestat injection exerted no effect on allcause mortality or ventilator-free days in patients with heterogeneous acute lung injury. ${ }^{32}$ One reason for this loss of effect of NE inhibition on neutrophil function could be related to tissue penetration. If sivelestat can be delivered selectively to injury sites by using new drug-delivery systems in the future, sivelestat administration might emerge as an effective clinical treatment against sepsis.

Sepsis is an extremely complicated disease compared with simple endotoxemia in an experimental model. Because our focus here was to investigate the direct relationship between endothelial glycocalyx injury and neutrophils, we used an endotoxemia model that does not reflect certain typical septic conditions such as bacterial infection, and this represents one limitation of our study. A previous study showed that $\mathrm{KO}$ mice that lack neutrophil elastase have impaired host defenses to Gram-negative bacteria, ${ }^{33}$ although the present study showed that neutrophil elastase was deleterious to the host. It is thought that the difference may be due to the presence or absence of bacteria. Neutrophils are one of the first responders of inflammatory cells and work against acute inflammation as a result of bacterial infection. Neutrophil elastase injures the endothelium through the endothelial glycocalyx while also requiring innate immunity to successfully battle a bacterial infection. Therefore, innate immunity may not be sufficient in the bacteremia model because G-CSFKO mice have depleted neutrophils. In other words, the neutrophil has two roles. In the present study, it was not confirmed whether neutrophil elastase altered endothelial glycocalyx directly. Additional studies that use a bacteremia model to further explore the role of endothelial glycocalyx in endothelial injury during sepsis are needed.

Another limitation of this study is that we did not examine inflammatory cytokines such as IL-6; the serum concentration of IL-6 in particular was reported to contribute to the survival rate in sepsis. ${ }^{34}$ In addition, the aforementioned report suggested that the endothelial injury 
was caused by several factors, including superoxides. The present study has not analyzed the influences of superoxides in endotoxemia mice. ${ }^{35}$

\section{Conclusion}

NE plays a pivotal role in pulmonary endothelial glycocalyx degradation; thus, inhibition of NE might offer a potential means to protect the endothelial glycocalyx.

\section{Acknowledgments}

K.S. and H.O. wrote the manuscript; G.T., C.T., and N.M. performed transmission electron microscopy (TEM) imaging; K.S., H.O., and K.O. performed scanning electron microscopy (SEM) imaging; C.T. prepared samples for TEM imaging; K.S., H.O., C.T., A.K., H.Y., and R.Z. prepared samples for SEM imaging; K.S., K.M., and H.T. performed immunohistochemical analysis; K.S. and T.F. performed the enzyme-linked immunosorbent assay study; K.S., H.O., A.K., H.Y., R.Z., S.M., A.U., S.S., T.K., T.W., T.Y., H.U., and S.Y. conducted the animal studies; K.S., A.K., S.S., and Y.M. performed the fluorescence-activated cell sorting analysis; S.O. supervised the animal studies; H.O. and G.T. revised and edited the manuscript; all authors read and approved the final manuscript.

\section{References}

1. Singer M, Deutschman CS, Seymour CW, Shankar-Hari M, Annane D, Bauer M, Bellomo R, Bernard GR, Chiche JD, Coopersmith CM, Hotchkiss RS, Levy MM, Marshall JC, Martin GS, Opal SM, Rubenfeld GD, van der Poll T, Vincent JL, Angus DC: The Third International Consensus Definitions for Sepsis and Septic Shock (Sepsis-3). JAMA 2016, 315:801-810

2. Iscimen R, Cartin-Ceba R, Yilmaz M, Khan H, Hubmayr RD, Afessa B, Gajic O: Risk factors for the development of acute lung injury in patients with septic shock: an observational cohort study. Crit Care Med 2008, 36:1518-1522

3. Schmidt EP, Yang Y, Janssen WJ, Gandjeva A, Perez MJ, Barthel L, Zemans RL, Bowman JC, Koyanagi DE, Yunt ZX, Smith LP, Cheng SS, Overdier KH, Thompson KR, Geraci MW, Douglas IS, Pearse DB, Tuder RM: The pulmonary endothelial glycocalyx regulates neutrophil adhesion and lung injury during experimental sepsis. Nat Med 2012, 18:1217-1223

4. Schmidt EP, Li G, Li L, Fu L, Yang Y, Overdier KH, Douglas IS, Linhardt RJ: The circulating glycosaminoglycan signature of respiratory failure in critically ill adults. J Biol Chem 2014, 289: 8194-8202

5. Inagawa R, Okada H, Takemura G, Suzuki K, Takada C, Yano H, Ando Y, Usui T, Hotta Y, Miyazaki N, Tsujimoto A, Zaikokuji R, Matsumoto A, Kawaguchi T, Doi T, Yoshida T, Yoshida S, Kumada K, Ushikoshi H, Toyoda I, Ogura S: Ultrastructural alteration of pulmonary capillary endothelial glycocalyx during endotoxemia. Chest 2018, 154:317-325

6. Chelazzi C, Villa G, Mancinelli P, De Gaudio AR, Adembri C: Glycocalyx and sepsis-induced alterations in vascular permeability. Crit Care 2015, 19:26
7. Manicone AM: Role of the pulmonary epithelium and inflammatory signals in acute lung injury. Expert Rev Clin Immunol 2009, 5:63-75

8. Abraham E: Neutrophils and acute lung injury. Crit Care Med 2003, 31:S195-S199

9. Hagiwara S, Iwasaka H, Togo K, Noguchi T: A neutrophil elastase inhibitor, sivelestat, reduces lung injury following endotoxininduced shock in rats by inhibiting HMGB1. Inflammation 2008, 31:227-234

10. Vender RL: Therapeutic potential of neutrophil-elastase inhibition in pulmonary disease. J Investig Med 1996, 44:531-539

11. Weiss SJ: Tissue destruction by neutrophils. N Engl J Med 1989, 320: 365-376

12. Lee JM, Yeo CD, Lee HY, Rhee CK, Kim IK, Lee DG, Lee SH, Kim JW: Inhibition of neutrophil elastase contributes to attenuation of lipopolysaccharide-induced acute lung injury during neutropenia recovery in mice. J Anesth 2017, 31:397-404

13. Miyazaki Y, Inoue T, Kyi M, Sawada M, Miyake S, Yoshizawa Y: Effects of a neutrophil elastase inhibitor (ONO-5046) on acute pulmonary injury induced by tumor necrosis factor alpha (TNFalpha) and activated neutrophils in isolated perfused rabbit lungs. Am J Respir Crit Care Med 1998, 157:89-94

14. Passi A, Negrini D, Albertini R, De Luca G, Miserocchi G: Involvement of lung interstitial proteoglycans in development of hydraulic- and elastase-induced edema. Am J Physiol 1998, 275: L631-L635

15. Committee for the Update of the Guide for the Care and Use of Laboratory Animals; National Research Council: Guide for the Care and Use of Laboratory Animals: Eighth Edition. Washington, DC, National Academies Press, 2011

16. Morishita K, Takemura G, Tsujimoto A, Kanamori H, Okada H, Chousa M, Ushimaru S, Mikami A, Kawamura I, Takeyama T, Kawaguchi T, Watanabe T, Goto K, Morishita M, Ushikoshi H, Kawasaki M, Ogura S, Minatoguchi S: Postinfarction cardiac remodeling proceeds normally in granulocyte colony-stimulating factor knockout mice. Am J Pathol 2015, 185:1899-1911

17. Suzuki K, Inoue S, Kametani Y, Komori Y, Chiba S, Sato T, Inokuchi S, Ogura S: Reduced immunocompetent B cells and increased secondary infection in elderly patients with severe sepsis. Shock 2016, 46:270-278

18. Langlois RA, Meyerholz DK, Coleman RA, Cook RT, Waldschmidt TJ, Legge KL: Oseltamivir treatment prevents the increased influenza virus disease severity and lethality occurring in chronic ethanol consuming mice. Alcohol Clin Exp Res 2010, 34: $1425-1431$

19. Okada H, Takemura G, Suzuki K, Oda K, Takada C, Hotta Y, Miyazaki N, Tsujimoto A, Muraki I, Ando Y, Zaikokuji R, Matsumoto A, Kitagaki H, Tamaoki Y, Usui T, Doi T, Yoshida T, Yoshida S, Ushikoshi H, Toyoda I, Ogura S: Three-dimensional ultrastructure of capillary endothelial glycocalyx under normal and experimental endotoxemic conditions. Crit Care 2017, 21:261

20. Lieschke GJ, Grail D, Hodgson G, Metcalf D, Stanley E, Cheers C, Fowler KJ, Basu S, Zhan YF, Dunn AR: Mice lacking granulocyte colony-stimulating factor have chronic neutropenia, granulocyte and macrophage progenitor cell deficiency, and impaired neutrophil mobilization. Blood 1994, 84:1737-1746

21. Imaki K, Okada T, Nakayama Y, Nagao Y, Kobayashi K, Sakai Y, Mohri T, Amino T, Nakai H, Kawamura M: Non-peptidic inhibitors of human neutrophil elastase: the design and synthesis of sulfonanilidecontaining inhibitors. Bioorg Med Chem 1996, 4:2115-2134

22. Weinbaum S, Tarbell JM, Damiano ER: The structure and function of the endothelial glycocalyx layer. Annu Rev Biomed Eng 2007, 9: $121-167$

23. Ando Y, Okada H, Takemura G, Suzuki K, Takada C, Tomita H, Zaikokuji R, Hotta Y, Miyazaki N, Yano H, Muraki I, Kuroda A, Fukuda H, Kawasaki Y, Okamoto H, Kawaguchi T, Watanabe T, Doi T, Yoshida T, Ushikoshi H, Yoshida S, Ogura S: Brainspecific ultrastructure of capillary endothelial glycocalyx and its 
possible contribution for blood brain barrier. Sci Rep 2018, 8: 17523

24. Wiener-Kronish JP, Albertine KH, Matthay MA: Differential responses of the endothelial and epithelial barriers of the lung in sheep to Escherichia coli endotoxin. J Clin Invest 1991, 88:864-875

25. Wiggs BR, English D, Quinlan WM, Doyle NA, Hogg JC, Doerschuk CM: Contributions of capillary pathway size and neutrophil deformability to neutrophil transit through rabbit lungs. J Appl Physiol (1985) 1994, 77:463-470

26. Grommes J, Soehnlein O: Contribution of neutrophils to acute lung injury. Mol Med 2011, 17:293-307

27. Spight D, Trapnell B, Zhao B, Berclaz P, Shanley TP: Granulocytemacrophage-colony-stimulating factor-dependent peritoneal macrophage responses determine survival in experimentally induced peritonitis and sepsis in mice. Shock 2008, 30:434-442

28. Stephens DP, Fisher DA, Currie BJ: An audit of the use of granulocyte colony-stimulating factor in septic shock. Intern Med J 2002, $32: 143-148$

29. Donnelly SC, MacGregor I, Zamani A, Gordon MW, Robertson CE, Steedman DJ, Little K, Haslett C: Plasma elastase levels and the development of the adult respiratory distress syndrome. Am J Respir Crit Care Med 1995, 151:1428-1433
30. Zemans RL, Colgan SP, Downey GP: Transepithelial migration of neutrophils: mechanisms and implications for acute lung injury. Am J Respir Cell Mol Biol 2009, 40:519-535

31. Li G, Jia J, Ji K, Gong X, Wang R, Zhang X, Wang H, Zang B: The neutrophil elastase inhibitor, sivelestat, attenuates sepsis-related kidney injury in rats. Int J Mol Med 2016, 38:767-775

32. Zeiher BG, Artigas A, Vincent JL, Dmitrienko A, Jackson K, Thompson BT, Bernard G; STRIVE Study Group: Neutrophil elastase inhibition in acute lung injury: results of the STRIVE study. Crit Care Med 2004, 32:1695-1702

33. Belaaouaj A, McCarthy R, Baumann M, Gao Z, Ley TJ, Abraham SN, Shapiro SD: Mice lacking neutrophil elastase reveal impaired host defense against gram negative bacterial sepsis. Nat Med 1998, 4:615-618

34. Iwamura H, Sato M, Wakitani K: Comparative study of glucocorticoids, cyclosporine A, and JTE-607 [(-)-Ethyl-N[3,5-dichloro2-hydroxy-4-[2-(4-methylpiperazin-1-yl)ethoxy]benzoyl]-L-phenylalaninate dihydrochloride] in a mouse septic shock model. J Pharmacol Exp Ther 2004, 311:1256-1263

35. Adachi T, Fukushima T, Usami Y, Hirano K: Binding of human xanthine oxidase to sulphated glycosaminoglycans on the endothelialcell surface. Biochem J 1993, 289(Pt 2):523-527 